\title{
Selbstmedikation
}

\section{Ärzte: Patienten können für OTC ruhig zahlen}

\author{
Dass die gesetzlichen Kassen rezeptfreie Arzneimittel weitgehend \\ nicht mehr zahlen, stößt bei Ärzten auf steigende Akzeptanz. Sie \\ betonen die finanzielle Eigenverantwortung der Patienten.
}

$\mathrm{n}$ einer nicht repräsentativen Befragung der „Ärzte Zeitung“, an der sich knapp 380 Leser beteiligt haben, wurde nun zum zweiten Mal untersucht, inwieweit sich die Haltung niedergelassener Ärzte zum Grünen Rezept im Vergleich zum Vorjahr verändert hat. Im Januar 2009 hatten 684 Leser bereits zu gleichlautenden Fragen Stellung genommen, die nun erneut Basis der Befragung gewesen sind.

Dass die Kassen seit 2004 rezeptfreie Arzneien nur noch in Ausnahmefällen bezahlen, trifft bei $71 \%$ der Antwortenden auf grundsätzliches Verständnis. Sie halten es im Grundsatz für richtig, dass der Gesetzgeber die finanzielle Eigenverantwortung der Patienten gestärkt hat. Bei der ersten Befragung im vergangenen Jahr waren nur $64,4 \%$ dieser Ansicht. Knapp 26\% (vorher $32,2 \%$ ) lehnen in der aktuellen Umfrage die Herausnahme von nicht verschreibungspflichtigen Medikamenten (,over the counter", OTC) aus dem Leistungskatalog ab mit der Begründung, dies schränke ihr Therapiespektrum ein.

Die Einschätzungen der teilnehmenden Leser gehen bei dieser Frage weit auseinander. Der Allgemeinarzt Matthias Seusing aus Kiel findet die Herausnahme von OTC „im Grundsatz gut“, moniert aber, der Gesetzgeber sei bei dieser "Aktion mit der Heckenschere" vorgegangen. Die Kinder- und Jugendärztin Dipl.-Med. Christina Gerth aus Colditz hat besonders Kinder und Jugendliche über zwölf Jahren im Blick. Diese Gruppe habe kein eigenes Einkommen, ,und
Eltern sind oft nicht bereit, die OTCArzneimittel zu bezahlen", klagt sie. Der Internist Dr. Hans-Hendrik Knäbchen aus Marienberg wiederum moniert, die Anwendung der Ausnahmeregeln sei oft kompliziert.

Anders beurteilt wird die Frage vom Allgemeinmediziner Andreas Hahn aus Poppenhausen. Er freut sich, dass er sich wenigstens bei rezeptfreien Arzneien „bei voller Therapiefreiheit keine RegressSorgen machen" muss. Und sein Kollege, der Allgemeinarzt Dr. Siegbert Müller aus Bruchsal, fordert schlichtweg: „Es sollte keine Ausnahmen geben." Weitgehend unverändert geblieben ist die Einschätzung der Ärzte dazu, welchen Stellenwert OTC in der Verordnungspraxis haben. Für 35,5\% der Antwortenden ist der Stellenwert hoch (2009 waren es $38,4 \%$ ), $62 \%$ (vorher 58,6\%) messen rezeptfreien Arzneien eine mittlere Bedeutung zu. Lediglich 5,3\% (zuvor $3,9 \%$ ) beurteilen die OTC-Arzneien als wenig oder überhaupt nicht wichtig in ihrem Verordnungsalltag. eb 